Discrete Comput Geom 33:403-421 (2005)

DOI: $10.1007 / \mathrm{s} 00454-004-1104-8$

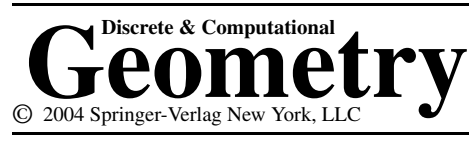

\title{
The Mixed Volume of Two Finite Vector Sets*
}

\author{
Li Xiaoyan and Leng Gangsong \\ Department of Mathematics, Hunan Normal University, \\ Changsha 410081, People's Republic of China \\ and \\ Department of Mathematics, Shanghai University, \\ Shanghai 200436, People's Republic of China \\ lixy77@sohu.com, lenggangsong@163.com
}

\begin{abstract}
We introduce the concept of the mixed volume of two finite vector sets in $R^{n}$. By employing the exterior differential, we prove a new and powerful inequality and establish a series of quantity relations associated with the mixed volume of two finite vector sets. As applications, we discuss some well-known results of simplices and the Hadamard inequality.
\end{abstract}

\section{Introduction}

The concept of mixed volumes, which forms a central part of the Bruun-Minkowski theory of convex bodies, arises naturally if one combines the two fundamental concepts of Minkowski addition and volume (see [23]). The theory of mixed volumes provides a unified treatment of various important metric quantities in geometry, such as volume, surface area, and mean width. The mixed volumes and the relatively new dual mixed volumes introduced by Lutwak (see [18]) have provided many powerful tools for solving problems involving projections and sections of convex bodies. They have also played an important role in isoperimetric problems in geometric analysis (see [6], [7], [18], [19], and [27]).

In this paper we give the definition of the mixed volume of two finite vector sets, which is the discrete form of a mixed volume. As its special case, we introduce the concepts of the mixed Cayley-Menger determinant and the mixed vertex angles of simplices, which are extensions of the well-known Cayley-Menger determinant and Bartoš vertex angles, respectively. In this paper we establish a series of inequalities and equalities

* This research was supported by National Natural Sciences Foundation of China (10271071). 
for the previously stated three new concepts, most of them are generalizations of some well-known results.

This paper, excluding the Introduction, is divided into four sections. In Section 2 we introduce the new concept of the mixed volume of two finite vector sets. By employing the exterior differential, we prove a powerful inequality which is analogous to the Minkowski inequality for mixed volumes of convex bodies, and, furthermore, study the dispersion of this inequality. In Section 3 we define the mixed Cayley-Menger determinant involving two simplices, and prove some new results associated with the two simplices. In Section 4 we introduce the concept of the mixed vertex angles of two simplices. We study the relationships between the vertex angles of simplices and the mixed volume of vector sets, and establish a number of new inequalities of the mixed vertex angles. Meanwhile, we obtain a new and simple proof of the well-known sine theorem. In Section 5 we reprove the inverse forms of the Hadamard inequality by using a new method, and show a series of new results for parallelotopes.

\section{The Mixed Volume of Two Finite Vector Sets}

In this section we introduce the concept of the mixed volume of two finite vector sets.

Let $X=\left\{x_{1}, x_{2}, \ldots, x_{k}\right\}$ be a vector set from $R^{n}$, and let $\Pi_{[X]}$ be the $k$-parallelotope which has $\left\{x_{1}, x_{2}, \ldots, x_{k}\right\}$ as $k$ edge vectors, and which lies in a $k$-hyperplane $\pi_{[X]}$. Let $V_{[X]}$ denote the $k$-dimensional volume of $\Pi_{[X]}$. In particular, when $k=n$, then $\pi_{[X]}=R^{n}$.

Definition 1. Let $X=\left\{x_{1}, x_{2}, \ldots, x_{k}\right\}$ and $Y=\left\{y_{1}, y_{2}, \ldots, y_{k}\right\}(k \in N)$ be two vector sets from $R^{n}$. The mixed volume $V_{[X, Y]}$ of $X$ and $Y$ can be defined by

$$
V_{[X, Y]}=\sqrt{\left|\operatorname{det}\left(\left\langle x_{\alpha}, y_{\beta}\right\rangle\right)_{1 \leq \alpha, \beta \leq k}\right|} .
$$

Obviously, the mixed volume $V_{[X, Y]}$ of two finite vector sets $X$ and $Y$ have following properties:

(1) $V_{[X, X]}=V_{[X]}$.

(2) $V_{\left[\lambda_{1} X, \lambda_{2} Y\right]}=\left|\lambda_{1} \lambda_{2}\right|^{k / 2} V_{[X, Y]}$, for $\lambda_{1}, \lambda_{2} \in R$.

(3) $V_{\left[\left\{x_{1}+x_{1}^{\prime}, x_{2}, \ldots, x_{k}\right\}, Y\right]}^{2} \leq V_{[X, Y]}^{2}+V_{\left[X^{\prime}, Y\right]}^{2}$, where $X^{\prime}=\left\{x_{1}^{\prime}, x_{2}, \ldots, x_{k}\right\}$.

(4) $V_{\left[\left\{x_{1}+x, x_{2}+x, \ldots, x_{k}+x\right\}, Y\right]}^{2} \leq V_{[X, Y]}^{2}+\sum_{i=1}^{n} V_{\left[X_{i}, Y\right]}^{2}$, where $X_{i}=\left\{x_{1}, \ldots, x_{i-1}, x\right.$, $\left.x_{i+1}, \ldots, x_{k}\right\}$.

(5) If $X$ or $Y$ is a linearly dependent vector set from $R^{n}$, it follows that $V_{[X, Y]}=0$. In particular, when $k>n$, we have $V_{[X, Y]}=0$.

According to the definition of $V_{[X, Y]}$, by applying the exterior differential, we obtain the following inequality which is analogous to the Minkowski inequality of mixed volumes of convex bodies.

Theorem 2.1. Suppose that $X=\left\{x_{1}, x_{2}, \ldots, x_{k}\right\}$ and $Y=\left\{y_{1}, y_{2}, \ldots, y_{k}\right\}(1 \leq k \leq n)$ are two linearly independent vector sets from $R^{n}$, respectively. Then

$$
V_{[X, Y]}^{2} \leq V_{[X]} V_{[Y]}
$$


with equality holds if and only if the hyperplane $\pi_{[X]} \| \pi_{[Y]}$. In particular, when $k=n$, the equality holds.

Proof. Let $e_{1}, e_{2}, \ldots, e_{n}$ be a standard orthogonal basis in $R^{n}$. Then

$$
\left\{e_{i_{1}} \wedge e_{i_{2}} \wedge \cdots \wedge e_{i_{k}}: 1 \leq i_{1}<\cdots<i_{k} \leq n\right\}
$$

is a standard orthogonal basis of $\bigwedge^{k} R^{n}$.

Since $x_{1} \wedge \cdots \wedge x_{k}, y_{1} \wedge \cdots \wedge y_{k} \in \wedge^{k} R^{n}$, let

$$
\left\langle x_{1} \wedge \cdots \wedge x_{k}, y_{1} \wedge \cdots \wedge y_{k}\right\rangle=\operatorname{det}\left(\left\langle x_{\alpha}, y_{\beta}\right\rangle\right)_{1 \leq \alpha<\beta \leq k} .
$$

Then $\langle$,$\rangle is a bilinear form in the space \bigwedge^{k} R^{n}$. It is easy to prove that $\langle$,$\rangle is the$ Euclidean inner product in $\bigwedge^{k} R^{n}$.

Since

$$
\left\|x_{1} \wedge \cdots \wedge x_{k}\right\|=V_{[X]}, \quad\left\|y_{1} \wedge \cdots \wedge y_{k}\right\|=V_{[Y]}
$$

and

$$
\left|\left\langle x_{1} \wedge \cdots \wedge x_{k}, y_{1} \wedge \cdots \wedge y_{k}\right\rangle\right|=V_{[X, Y]}^{2},
$$

by applying the Cauchy inequality to the above two equalities, (2.1) follows.

When $k<n$, it is easy to see the equality of (2.1) if and only if

$$
x_{1} \wedge \cdots \wedge x_{k}=\lambda y_{1} \wedge \cdots \wedge y_{k}
$$

where $\lambda \neq 0$ is a constant, equivalently, $\pi_{[X]} \| \pi_{[Y]}$.

When $k=n$, the space $\bigwedge^{k} R^{n}$ is one-dimensional, it implies that the equality of (2.1) holds.

To estimate the difference of $V_{[X, Y]}^{2}$ and $V_{[X]} V_{[Y]}$, we define the dispersion by

$$
\delta=V_{[X]} V_{[Y]}-V_{[X, Y]}^{2} .
$$

Obviously, we have

(1) $\delta \geq 0$

(2) If $\pi_{[X]} \| \pi_{[Y]}$, then $\delta=0$.

For the dispersion of inequality (2.1), we have the following result.

Theorem 2.2 Suppose that $X=\left\{x_{1}, x_{2}, \ldots, x_{k}\right\}$ and $Y=\left\{y_{1}, y_{2}, \ldots, y_{k}\right\}(1 \leq k \leq n)$ are two vector sets from $R^{n}$, respectively, and $u, v, p, q$ are real non-negative numbers with $q \leq p$. If

$$
p V_{[X]}^{2}<u^{2} \quad \text { and } \quad p V_{[Y]}^{2}<v^{2}
$$


then

$$
(p-q) \delta \geq\left(u^{2}-p V_{[X]}^{2}\right)^{1 / 2}\left(v^{2}-p V_{[Y]}^{2}\right)^{1 / 2}+p V_{[X]} V_{[Y]}-u v
$$

with equality holds if and only if the hyperplane $\pi_{[X]} \| \pi_{[Y]}$ and $v V_{[X]}=u V_{[Y]}$.

Proof. Since

$$
u^{2}-(p-q) V_{[X]}^{2} \geq u^{2}-p V_{[X]}^{2}>0
$$

let

$$
\begin{aligned}
f(x) & =\left[u^{2}-(p-q) V_{[X]}^{2}\right] x^{2}-2\left[u v-(p-q) V_{[X]} V_{[Y]}\right] x+\left[v^{2}-(p-q) V_{[Y]}^{2}\right] \\
& =(u x-v)^{2}-(p-q)\left(x V_{[X]}-V_{[Y]}\right)^{2}
\end{aligned}
$$

According to the above suppose we have $u \neq 0$, furthermore,

$$
f\left(\frac{v}{u}\right)=-(p-q)\left(\frac{v}{u} V_{[X]}-V_{[Y]}\right)^{2} \leq 0
$$

Since, $f(x) \rightarrow+\infty \quad(x \rightarrow+\infty)$ and $f(x) \rightarrow+\infty \quad(x \rightarrow-\infty)$, therefore, $f(x)$ has two different real zeros belonging to $(-\infty, v / u)$ and $(v / u,+\infty)$ respectively, or, has a zero of order 2, i.e., $x_{1,2}=v / u$. Hence, the discriminant of the above quadratic (2.3) is non-negative, i.e.,

$$
\left[u^{2}-(p-q) V_{[X]}^{2}\right]\left[v^{2}-(p-q) V_{[Y]}^{2}\right] \leq\left[u v-(p-q) V_{[X]} V_{[Y]}\right]^{2}
$$

Since $u v-(p-q) V_{[X]} V_{[Y]}>0$, and using Theorem 2.1, we have that

$$
\left[u^{2}-(p-q) V_{[X]}^{2}\right]^{1 / 2}\left[v^{2}-(p-q) V_{[Y]}^{2}\right]^{1 / 2} \leq u v-(p-q) V_{[X, Y]}^{2} .
$$

On the other hand, implying the Cauchy inequality, we obtain

$$
\begin{gathered}
{\left[u^{2}-(p-q) V_{[X]}^{2}\right]^{1 / 2}\left[v^{2}-(p-q) V_{[Y]}^{2}\right]^{1 / 2}} \\
\quad=\left[\left(u^{2}-p V_{[X]}^{2}\right)+q V_{[X]}^{2}\right]^{1 / 2}\left[\left(v^{2}-p V_{[Y]}^{2}\right)+q V_{[Y]}^{2}\right]^{1 / 2} \\
\geq\left(u^{2}-p V_{[X]}^{2}\right)^{1 / 2}\left(v^{2}-p V_{[Y]}^{2}\right)^{1 / 2}+q V_{[X]} V_{[Y]} .
\end{gathered}
$$

Combining (2.4) and (2.5), we get that

$$
u v-p V_{[X, Y]}^{2}+q V_{[X, Y]}^{2} \geq\left(u^{2}-p V_{[X]}^{2}\right)^{1 / 2}\left(v^{2}-p V_{[Y]}^{2}\right)^{1 / 2}+q V_{[X]} V_{[Y]}
$$

Rearranging (2.6), the desired (2.2) follows.

From the conditions of equalities (2.4) and (2.5), we obtain that equality (2.2) holds if and only if the hyperplane $\pi_{[X]} \| \pi_{[Y]}$ and $v V_{[X]}=u V_{[Y]}$. 
When $q=0$, we get the following inequality which is analogous to the Aczél inequality.

Corollary 1. Under the hypotheses of Theorem 2.2, we have that

$$
\left(u^{2}-p V_{[X]}^{2}\right)\left(v^{2}-p V_{[Y]}^{2}\right) \leq\left(u v-p V_{[X, Y]}^{2}\right)^{2}
$$

with equality holds if and only if the hyperplane $\pi_{[X]} \| \pi_{[Y]}$ and $v V_{[X]}=u V_{[Y]}$.

\section{Some Results Involving Two Simplices}

The quantity relations involving two simplices have been an interesting subject. The well-known Neuberg-Pedoe inequality is the first inequality involving two triangles [20]. Following Pedoe, a number of inequalities for two simplices have been established (see [15], [21], and [29]). Yang and Zhang in [28] generalized the Neuberg-Pedoe inequality to $R^{n}$. In [28] Yang and Zhang effectively applied the well-known Cayley-Menger determinant to obtain their elegant result.

Let $\mathcal{A}=\left\{A_{0}, A_{1}, \ldots, A_{m}\right\}(m \geq n)$ be a set of points in $R^{n}$, and let $\rho_{i j}=$ $\left\|A_{i} A_{j}\right\|(0 \leq i, j \leq m)$, then the Cayley-Menger determinant (see [2]) of $\mathcal{A}$ is defined by

$$
M(\mathcal{A})=\left|\begin{array}{ccccc}
0 & 1 & 1 & \cdots & 1 \\
1 & 0 & \rho_{01}^{2} & \cdots & \rho_{0 m}^{2} \\
1 & \rho_{10}^{2} & 0 & \cdots & \rho_{1 m}^{2} \\
\vdots & \vdots & \vdots & \cdots & \vdots \\
1 & \rho_{m 1}^{2} & \rho_{m 2}^{2} & \cdots & 0
\end{array}\right| .
$$

When $\mathcal{A}$ is the vertex set of an $n$-simplex (i.e., $m=n$ ) with volume $V$, there is a famous result which combines the Cayley-Menger determinant with the volume of simplex as follows (see [28]):

$$
V^{2}=\frac{(-1)^{n} M(\mathcal{A})}{2^{n+1} n !^{2}}
$$

Now we introduce the mixed Cayley-Menger determinant of two point sets as follows.

Definition 2. Suppose $\mathcal{A}=\left\{A_{0}, A_{1}, \ldots, A_{m}\right\}$ and $\mathcal{B}=\left\{B_{0}, B_{1}, \ldots, B_{m}\right\} \quad(m \geq n)$ are two point sets in $R^{n}$ and $d_{i j}=\overrightarrow{A_{i} B_{j}}(0 \leq i, j \leq m)$. Let

$$
M(\mathcal{A}, \mathcal{B})=\left|\begin{array}{llll}
0 & 1 & \ldots & 1 \\
1 & & \\
\vdots & & d_{i j}^{2} & \left.\right|_{0 \leq i, j \leq m} . \\
1 & & &
\end{array}\right|
$$

Then $M(\mathcal{A}, \mathcal{B})$ is called the mixed Cayley-Menger determinant of the point sets $\mathcal{A}$ and $\mathcal{B}$. 


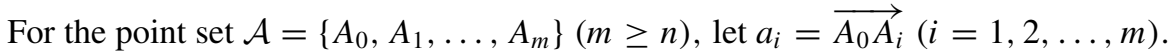
We call $X=\left\{a_{1}, a_{2}, \ldots, a_{m}\right\}$ the edge-vector set of $\mathcal{A}$ at $A_{0}$. There is a relationship between the mixed Cayley-Menger determinant $M(\mathcal{A}, \mathcal{B})$ and the mixed volume of two edge-vector sets as follows.

Theorem 3.1. Let $M(\mathcal{A}, \mathcal{B})$ be the mixed Cayley-Menger determinant of two point sets $\mathcal{A}=\left\{A_{0}, A_{1}, \ldots, A_{m}\right\}$ and $\mathcal{B}=\left\{B_{0}, B_{1}, \ldots, B_{m}\right\}(m \geq n)$ in $R^{n}$, let $X$ and $Y$ be two edge-vector sets of $\mathcal{A}$ at $A_{0}$ and $\mathcal{B}$ at $B_{0}$, respectively, and let $V_{[X, Y]}$ be the mixed volume of $X$ and $Y$. Then

$$
|M(\mathcal{A}, \mathcal{B})|=2^{m+1} V_{[X, Y]}^{2} .
$$

Proof. For convenience, we denote $a_{0}=\overrightarrow{A_{0} A_{0}}$ and $b_{0}=\overrightarrow{B_{0} B_{0}}$. According to the definition of determinant $M(\mathcal{A}, \mathcal{B})$, we have

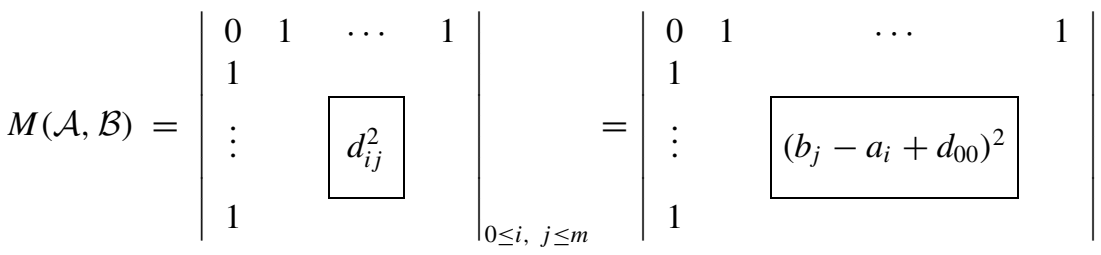

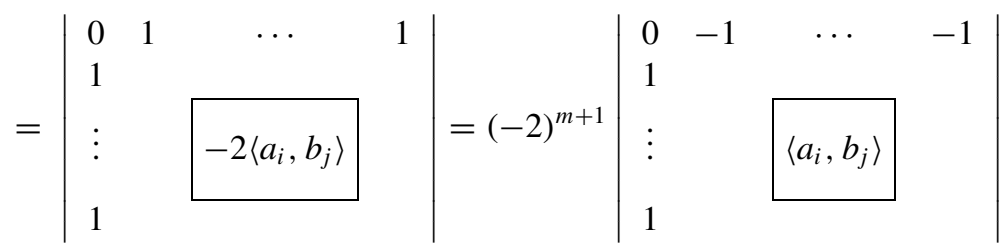

$$
\begin{aligned}
& =\operatorname{det}\left(\left\langle a_{i}, b_{j}\right\rangle\right)_{0 \leq i, j \leq m}+(-2)^{m+1}\left|\begin{array}{cccc}
0 & -1 & \cdots & -1 \\
1 & & & \\
\vdots & & & \\
1 & & & \left\langle a_{i}, b_{j}\right\rangle
\end{array}\right|
\end{aligned}
$$

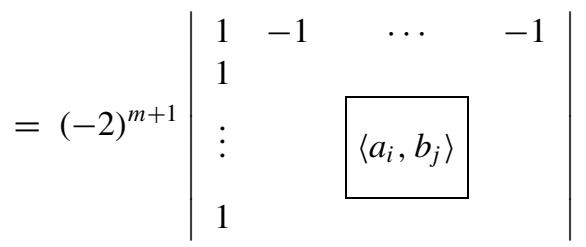

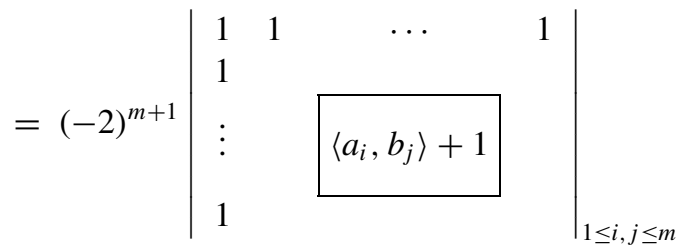




$$
\begin{aligned}
& =(-2)^{m+1}\left|\begin{array}{cccc}
1 & 0 & \cdots & 0 \\
1 & & \\
\vdots & & \left\langle a_{i}, b_{j}\right\rangle \\
1 &
\end{array}\right|_{1 \leq i, j \leq m} \\
& =(-2)^{m+1} \cdot \operatorname{det}\left(\left\langle a_{i}, b_{j}\right\rangle\right)_{1 \leq i, j \leq m} .
\end{aligned}
$$

According to the definition of the mixed volume $V_{[X, Y]}$, we can get (3.2).

Corollary 2. Under the hypotheses of Theorem 3.1, if $m \geq n+1$, then $M(\mathcal{A}, \mathcal{B})=0$.

From Theorem 3.1, and noting the volume formula of a simplex, we obtain the following theorem immediately.

Theorem 3.2. Let $\mathcal{A}=\left\langle A_{0}, A_{1}, \ldots, A_{n}\right\rangle$ and $\mathcal{B}=\left\langle B_{0}, B_{1}, \ldots, B_{n}\right\rangle$ be two $n$-simplices with the volumes $V_{\mathcal{A}}$ and $V_{\mathcal{B}}$ in $R^{n}$. Then

$$
|M(\mathcal{A}, \mathcal{B})|=2^{n+1} n !^{2} V_{\mathcal{A}} V_{\mathcal{B}} .
$$

Let $\mathcal{A}=\left\langle A_{0}, A_{1}, \ldots, A_{n}\right\rangle$ and $\mathcal{B}=\left\langle B_{0}, B_{1}, \ldots, B_{n}\right\rangle$ be two $n$-simplices in $R^{n}$ and let $d_{i j}=\vec{A}_{i} B_{j}(0 \leq i, j \leq n)$. Then the matrix

$$
D(\mathcal{A}, \mathcal{B})=\left(d_{i j}^{2}\right)_{(n+1) \times(n+1)}
$$

is called the mixed distance matrix of $\mathcal{A}$ and $\mathcal{B}$.

When $m=n$, let $\mathcal{A}=\left\langle A_{0}, A_{1}, \ldots, A_{n}\right\rangle$ be an $n$-simplex, and let $a_{i}=\overrightarrow{A_{0} A_{i}} \quad(i=$ $1, \ldots, n)$. Then $X=\left\{a_{1}, a_{2}, \ldots, a_{n}\right\}$ is the edge-vector set of the simplex $\mathcal{A}$ at vertex $A_{0}$.

Theorem 3.3. Let $R_{\mathcal{A}}$ and $R_{\mathcal{B}}$ be the circumradius of two $n$-simplices $\mathcal{A}$ and $\mathcal{B}$ in $R^{n}$, let $X$ and $Y$ be two edge-vector sets of $\mathcal{A}$ at $A_{0}$ and $\mathcal{B}$ at $B_{0}$, respectively, and let $V_{[X, Y]}$ be the mixed volume of $X$ and $Y$. Then

$$
|\operatorname{det} D(\mathcal{A}, \mathcal{B})|=\frac{1}{2^{n+1}}\left|R_{\mathcal{A}}^{2}+R_{\mathcal{B}}^{2}-d^{2}\right| V_{[X, Y]}^{2},
$$

where $d={\overrightarrow{O_{\mathcal{A}}}}_{\mathcal{B}}$, here, $O_{\mathcal{A}}$ and $O_{\mathcal{B}}$ are the circumcenters of $\mathcal{A}$ and $\mathcal{B}$, respectively.

Proof. Let $\mathbb{A}=\left\{O_{\mathcal{B}}, A_{0}, \ldots, A_{n}\right\}$ and $\mathbb{B}=\left\{O_{\mathcal{A}}, B_{0}, \ldots, B_{n}\right\}$ be two $(n+2)$ points sets. According to Corollary 2, we get

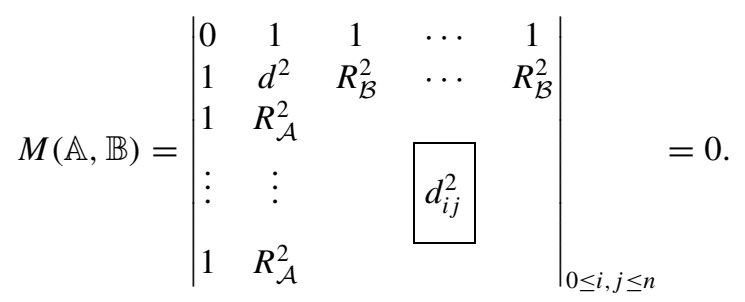


Using the properties of the determinant, we can get

$$
\left(d^{2}-R_{\mathcal{A}}^{2}-R_{\mathcal{B}}^{2}\right) M(\mathcal{A}, \mathcal{B})-\operatorname{det} D(\mathcal{A}, \mathcal{B})=0 .
$$

Applying Theorem 3.2, (3.4) follows.

Corollary 3. Let $\mathcal{A}$ and $\mathcal{B}$ be two $n$-simplices inscribing a unit sphere. Then

$$
\frac{|\operatorname{det} D(\mathcal{A}, \mathcal{B})|}{V_{[X, Y]}^{2}}=2^{n+2} .
$$

For a $k$-simplex $\mathcal{A}=\left\langle A_{0}, A_{1}, \ldots, A_{k}\right\rangle$ with $k$-dimensional volume $V$, let

$$
\delta_{i j}=\frac{1}{2}\left(\left\|A_{0} A_{i}\right\|^{2}+\left\|A_{0} A_{j}\right\|^{2}-\left\|A_{i} A_{j}\right\|^{2}\right) \quad(1 \leq i, j \leq k) .
$$

Then there is the following useful formula:

$$
V=\frac{1}{k !} \operatorname{det}\left(\delta_{i j}\right)_{1 \leq i, j \leq k} .
$$

Now we give a generalization of this formula involving two simplices.

Theorem 3.4. Let $\mathcal{A}=\left\langle A_{0}, A_{1}, \ldots, A_{k}\right\rangle$ and $\mathcal{B}=\left\langle A_{0}, B_{1}, \ldots, B_{k}\right\rangle$ be two k-simplices which have a common vertex $A_{0}$, with $k$-dimensional volumes $V_{\mathcal{A}}$ and $V_{\mathcal{B}}$, respectively. Put

$$
q_{i j}=\frac{1}{2}\left(\left\|A_{0} A_{i}\right\|^{2}+\left\|A_{0} B_{j}\right\|^{2}-\left\|A_{i} B_{j}\right\|^{2}\right) \quad i, j \in\{1,2, \ldots, k\} .
$$

Then

$$
\operatorname{det}\left(q_{i j}\right)_{k \times k} \leq k !^{2} V_{\mathcal{A}} V_{\mathcal{B}}
$$

with equality holds if and only if the hyperplane $\pi_{A_{k}} \| \pi_{B_{k}}$, where $\pi_{A_{k}}$ is the hyperplane containing $A_{i_{1}}, A_{i_{2}}, \ldots, A_{i_{k}}\left(0 \leq i_{1}, \ldots, i_{k} \leq k\right)$. In particular, when $k=n$, then

$$
\operatorname{det}\left(q_{i j}\right)_{n \times n}=n !^{2} V_{\mathcal{A}} V_{\mathcal{B}} .
$$

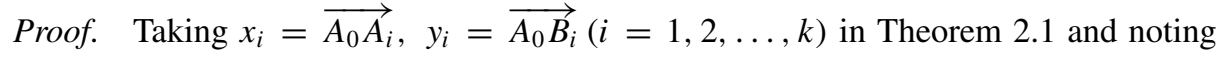
that

$$
\left\langle x_{i}, y_{j}\right\rangle=\left\|x_{i}\right\|\left\|y_{j}\right\| \cos \alpha_{i j}=q_{i j},
$$

where $\alpha_{i j}$ is the angle between $x_{i}$ and $y_{j}$, the desired (3.6) follows.

From the condition of Theorem 2.1, we obtain (3.7).

\section{The Vertex Angles of Simplices and the Sine Theorem}

For an $n$-simplex $\Omega=\left\langle A_{0}, A_{1}, \ldots, A_{n}\right\rangle$, let $\Omega_{i}=\left\langle A_{0}, \ldots, A_{i-1}, A_{i+1} \ldots, A_{n}\right\rangle$ be its $(n-1)$-dimensional facet whose area is $S_{i}$, with $h_{i}$ the altitude of $\Omega$ from the vertex $A_{i}(i=0,1, \ldots, n)$ and $\varphi_{i j}$ the internal dihedral angle between $\Omega_{i}$ and $\Omega_{j}$. Paper [1] introduces the concept of the vertex angle of a simplex. 
Bartoš in [1] gave the definition of the vertex angles of an $n$-dimensional simplex as follows:

Suppose that $\left\{e_{0}, e_{1}, \ldots, e_{n}\right\}$ is the set of the unit outer normal vector of facets of $\Omega$. The vertex angle at $A_{i}$ can be defined by

$$
\varphi_{i}=\arcsin \operatorname{det}\left(e_{0}, \ldots, e_{i-1}, e_{i+1}, \ldots, e_{n}\right) \quad(i=0,1, \ldots, n) .
$$

Bartoš established the following sine theorem based on his definition (see [1]).

\section{Theorem 4.1.}

$$
S_{0} \cdots S_{i-1} S_{i+1} \cdots S_{n} \sin \varphi_{i}=\frac{(n V)^{n-1}}{(n-1) !} \quad(i=0,1, \ldots, n) .
$$

The above sine theorem has reappeared several times in different forms and different proofs. Quite recently, it played an important role in the investigation of geometric inequalities (see [5], [14], and [24]).

In this section we give a new and simple proof of the sine theorem by applying Theorem 2.1.

Proof of Theorem 4.1. Let

$$
\begin{aligned}
X & =\left\{\overrightarrow{A_{i} A_{0}}, \ldots, \vec{A}_{i} \vec{A}_{i-1}, \vec{A}_{i} \vec{A}_{i+1}, \ldots, \overrightarrow{A_{i} A_{n}}\right\}, \\
Y & =\left\{e_{0}, \ldots, e_{i-1}, e_{i+1}, \ldots, e_{n}\right\} \quad(i=0,1, \ldots, n),
\end{aligned}
$$

noting that

$$
\left\langle\overrightarrow{A_{i} A_{j}}, e_{k}\right\rangle=\delta_{j k}\left( \pm h_{j}\right) \quad(j, k \neq i),
$$

where

$$
\delta_{j k}= \begin{cases}1, & j=k \\ 0, & j \neq k\end{cases}
$$

Then

$$
\begin{gathered}
V_{[X, Y]}^{2}=\left|\operatorname{det}\left(\left\langle\overrightarrow{A_{i} A_{j}}, e_{k}\right\rangle\right)_{j, k \neq i}\right|=h_{0} \cdots h_{i-1} h_{i+1} \cdots h_{n}, \\
V_{[X]} V_{[Y]}=n ! V \sin \varphi_{i} .
\end{gathered}
$$

Employing Theorem 2.1,

$$
n ! V \sin \varphi_{i}=h_{0} \cdots h_{i-1} h_{i+1} \cdots h_{n}=\frac{(n V)^{n}}{S_{0} \cdots S_{i-1} S_{i+1} \cdots S_{n}} .
$$

The proof is finished.

In [12] Leng and Zhang introduced the concept of $k$-order vertex angles of an $n$ simplex $\Omega=\left\langle A_{0}, A_{1}, \ldots, A_{n}\right\rangle$ in $R^{n}$ as follows: 
Suppose that $\left\{e_{0}, e_{1}, \ldots, e_{n}\right\}$ is the set of the unit outer normal vector of facets of $\Omega$. Let $e_{i_{1}}, e_{i_{2}}, \ldots, e_{i_{k}}\left(0 \leq i_{1}<i_{2}<\cdots<i_{k} \leq n\right)$ be $k$ vectors among this set. We call

$$
\theta_{i_{1} i_{2} \cdots i_{k}}=\arcsin \sqrt{\operatorname{det}\left(\left\langle e_{i_{r}}, e_{i_{s}}\right\rangle\right)_{k \times k}}, \quad i_{r}, i_{s} \in\left\{i_{1}, i_{2}, \ldots, i_{k}\right\},
$$

the $k$-order vertex angle at vertex set $\left\{A_{i_{1}}, A_{i_{2}}, \ldots, A_{i_{k}}\right\}$ of $\Omega$.

Paper [26] extended the concept of the $k$-order vertex angles of a simplex to two simplices, and introduced the concept of the $k$-order mixed vertex angles of two simplices.

Let $\Omega=\left\langle A_{0}, A_{1}, \ldots, A_{n}\right\rangle$ and $\Omega^{\prime}=\left\langle A_{0}^{\prime}, A_{1}^{\prime}, \ldots, A_{n}^{\prime}\right\rangle$ denote two $n$-simplices in $R^{n}$, and let $\Omega_{i}=\left\langle A_{0}, \ldots, A_{i-1}, A_{i+1}, \ldots, A_{n}\right\rangle$ and $\Omega_{i}^{\prime}=\left\langle A_{0}^{\prime}, \ldots, A_{i-1}^{\prime}, A_{i+1}^{\prime} \ldots, A_{n}^{\prime}\right\rangle$ be their $(n-1)$-dimensional facets $(i=0,1, \ldots, n)$, respectively.

Definition 3. Suppose that $\left\{e_{0}, e_{1}, \ldots, e_{n}\right\}$ and $\left\{e_{0}^{\prime}, e_{1}^{\prime}, \ldots, e_{n}^{\prime}\right\}$ are two sets of the unit outer normal vector of facets of two simplices $\Omega$ and $\Omega^{\prime}$, respectively. Let

$$
\tilde{\theta}_{i_{1} i_{2} \cdots i_{k}}=\arcsin \sqrt{\left|\operatorname{det}\left(\left\langle e_{i_{r}}, e_{i_{s}}^{\prime}\right\rangle\right)_{k \times k}\right|}, \quad i_{r}, i_{s} \in\left\{i_{1}, i_{2}, \ldots, i_{k}\right\} .
$$

We call $\tilde{\theta}_{i_{1} i_{2} \ldots i_{k}}$ the $k$-order mixed vertex angle at the vertex set $\left\{A_{i_{1}}, A_{i_{2}}, \ldots, A_{i_{k}}\right\}$ of $\Omega$ and the vertex set $\left\{A_{i_{1}}^{\prime}, A_{i_{2}}^{\prime}, \ldots, A_{i_{k}}^{\prime}\right\}$ of $\Omega^{\prime}$. In particular, $\widetilde{\varphi}_{i j}$ denotes the mixed internal dihedral angle between $\Omega_{i}$ and $\Omega_{j}^{\prime}$, and $\widetilde{\varphi}_{i}$ denotes the $n$-order mixed vertex angle at the vertices $A_{i}$ and $A_{i}^{\prime}$.

It is easy to see that the $k$-order mixed vertex angles are just $k$-order vertex angles when $\Omega=\Omega^{\prime}$.

For $k$-order mixed vertex angles, we establish the following inequalities.

\section{Theorem 4.2.}

$$
\sin ^{2} \widetilde{\theta}_{i_{1} i_{2} \cdots i_{k}} \leq \sin \theta_{i_{1} i_{2} \cdots i_{k}} \sin \theta_{i_{1} i_{2} \cdots i_{k}}^{\prime}
$$

with equality holds if and only if the hyperplane $\pi_{k} \| \pi_{k^{\prime}}$, where $\pi_{k}$ is the hyperplane containing $\left\{e_{i_{1}}, e_{i_{2}}, \ldots, e_{i_{k}}\right\}$.

Proof. Taking $x_{i}=e_{i}, y_{i}=e_{i}^{\prime}\left(i=i_{1}, i_{2}, \ldots, i_{k}\right)$ in Theorem 2.1, we obtain the proof of the theorem.

Using the known fact (see [8]),

$$
\prod_{0 \leq i_{1}<\cdots<i_{k} \leq n} \sin \theta_{i_{1} i_{2} \cdots i_{k}} \leq\left[\frac{(n+1)^{k}}{(k+1)^{n}}\right]^{((n+1) / n) C_{n}^{k}}
$$

with equality holds if and only if $\Omega$ is regular.

From (4.2) and the above inequality, we obtain the following corollary:

\section{Corollary 4.}

$$
\prod_{0 \leq i_{1}<\cdots<i_{k} \leq n} \sin \widetilde{\theta}_{i_{1} i_{2} \cdots i_{k}} \leq\left[\frac{(n+1)^{k}}{(k+1)^{n}}\right]^{((n+1) / n) C_{n}^{k}}
$$


with equality holds if and only if all the hyperplanes $\pi_{k} \| \pi_{k^{\prime}}$ and $\Omega$ and $\Omega^{\prime}$ are regular.

\section{Theorem 4.3.}

$$
\cos ^{2} \widetilde{\theta}_{i_{1} i_{2} \cdots i_{k}} \geq \cos \theta_{i_{1} i_{2} \cdots i_{k}} \cos \theta_{i_{1} i_{2} \cdots i_{k}}^{\prime}
$$

with equality holds if and only if the hyperplane $\pi_{k} \| \pi_{k^{\prime}}$ and $\theta_{i_{1} i_{2} \cdots i_{k}}=\theta_{i_{1} i_{2} \cdots i_{k}}^{\prime}$ or $\theta_{i_{1} i_{2} \ldots i_{k}}=\pi-\theta_{i_{1} i_{2} \ldots i_{k}}^{\prime}$.

Proof. Let $u=v=p=1, q=0$, then taking $x_{i}=e_{i}, y_{i}=e_{i}^{\prime}\left(i=i_{1}, i_{2}, \ldots, i_{k}\right)$ in Theorem 2.2, the desired proof of Theorem 4.3 is finished.

Theorem 4.4. Let $\widetilde{\theta}_{i_{1} i_{2} \ldots i_{k}}$ be a $k$-order mixed vertex angle of two $n$-simplices $\Omega$ and $\Omega^{\prime}$, and let $m_{0}, m_{1}, \ldots, m_{n}$ be $n+1$ real positive constants. Then

$$
\sum_{0 \leq i_{1}<\cdots<i_{k} \leq n}\left(\prod_{1 \leq j \leq k} m_{i_{j}}\right) \sin ^{2} \widetilde{\theta}_{i_{1} i_{2} \cdots i_{k}} \leq \frac{(n-1) !}{k !(n-k) ! n^{k-1}}\left(\sum_{i=0}^{n} m_{i}\right)^{k} .
$$

Equality (4.4) occurs if and only if all the hyperplanes $\pi_{k} \| \pi_{k^{\prime}}, \sin \theta_{i_{1} i_{2} \cdots i_{k}}=$ $c \sin \theta_{i_{1} i_{2} \ldots i_{k}}^{\prime}$ (where $\left.0 \leq c \leq 1\right)$, and all the following equalities hold:

$$
\frac{m_{t}}{\sum_{i=0}^{n} m_{i}}=\frac{\cos \varphi_{r s}}{n\left(\cos \varphi_{r s}+\cos \varphi_{r t} \cos \varphi_{s t}\right)}=\frac{\cos \varphi_{r s}^{\prime}}{n\left(\cos \varphi_{r s}^{\prime}+\cos \varphi_{r t}^{\prime} \cos \varphi_{s t}^{\prime}\right)}
$$

(where $0 \leq r, s, t \leq n$, and $r, s$, $t$ are pairwise unequal). In particular, the equality holds when all the hyperplanes $\pi_{k} \| \pi_{k^{\prime}}, \sin \theta_{i_{1} i_{2} \cdots i_{k}}=c \sin \theta_{i_{1} i_{2} \cdots i_{k}}^{\prime}$ (where $\left.0 \leq c \leq 1\right), \Omega$ and $\Omega^{\prime}$ are regular, and $m_{0}=m_{1}=\cdots=m_{n}$.

Proof. Let $A$ be the metric matrix of $\Omega$, that is,

$$
A=\left(\left\langle e_{i}, e_{j}\right\rangle\right)_{i, j=0}^{n}=\left(\begin{array}{cccc}
1 & & -\cos \varphi_{i j} & \\
& 1 & \ddots & \\
-\cos \varphi_{i j} & & 1
\end{array}\right)_{(n+1) \times(n+1)} .
$$

Then $A$ is a positive semi-definite symmetric matrix of rank $n$ [28].

Let

$$
C=\left(\begin{array}{cccc}
m_{0} & & -\sqrt{m_{i} m_{j}} \cos \varphi_{i j} & \\
& m_{1} & \ddots & \\
& & & m_{n}
\end{array}\right)_{(n+1) \times(n+1)} .
$$

Since the matrix $C$ has the form $D A D$ where $D$ is diagonal with diagonal entries $\sqrt{m_{i}}, C$ is a positive semi-definite symmetric matrix. It is easy to see that the rank of $C$ is $n$. So one of the eigenvalues of $C$ is zero, and the remaining $n$ eigenvalues are positive. Without loss of generality, we assume that $\lambda_{0}, \lambda_{1}, \ldots, \lambda_{n-1}$ are positive eigenvalues of $C$, and $\lambda_{n}$ is a zero eigenvalue of $C$. 
Let $E_{k}(C)$ be the sum of all the $k \times k$ principal minors of $C$, and let $S_{k}\left(\lambda_{0}, \lambda_{1}, \ldots, \lambda_{n}\right)$ be the $k$ th elementary symmetric polynomial of the eigenvalues $\lambda_{0}, \lambda_{1}, \ldots, \lambda_{n}$.

According to the fairly well-known fact

$$
E_{k}(C)=S_{k}\left(\lambda_{0}, \lambda_{1}, \ldots, \lambda_{n}\right)
$$

(see $(1.2,10)$ of [9]), Maclaurin's inequality, and noting $\lambda_{n}=0$, we have

$$
\begin{aligned}
& \sum_{0 \leq i_{1}<\cdots<i_{k} \leq n}\left(\prod_{1 \leq j \leq k} m_{i_{j}}\right) \sin ^{2} \theta_{i_{1} i_{2} \cdots i_{k}} \\
&=E_{k}(C)=S_{k}\left(\lambda_{0}, \lambda_{1}, \ldots, \lambda_{n}\right)=S_{k}\left(\lambda_{0}, \lambda_{1}, \ldots, \lambda_{n-1}\right) \\
& \leq {\left[S_{1}\left(\lambda_{0}, \lambda_{1}, \ldots, \lambda_{n-1}\right) /\left(\begin{array}{l}
n \\
1
\end{array}\right)\right]^{k}\left(\begin{array}{l}
n \\
k
\end{array}\right) } \\
&= {\left[S_{1}\left(\lambda_{0}, \lambda_{1}, \ldots, \lambda_{n}\right) /\left(\begin{array}{l}
n \\
1
\end{array}\right)\right]^{k}\left(\begin{array}{l}
n \\
k
\end{array}\right) } \\
&= {\left[E_{1}(C) /\left(\begin{array}{l}
n \\
1
\end{array}\right)\right]^{k}\left(\begin{array}{l}
n \\
k
\end{array}\right)=\left(\frac{1}{n} \sum_{i=1}^{n} m_{i}\right)^{k}\left(\begin{array}{l}
n \\
k
\end{array}\right) . }
\end{aligned}
$$

It is clear that the above equality holds if and only if

$$
\lambda_{0}=\lambda_{1}=\cdots=\lambda_{n-1}=\frac{1}{n} \operatorname{tr} C=\frac{1}{n} \sum_{i=0}^{n} m_{i}
$$

Let

$$
B=\left(\frac{1}{n} \sum_{i=0}^{n} m_{i}\right) I_{n+1}-C, \quad \text { where } \quad I_{n+1} \text { is an }(n+1) \times(n+1) \text { unit matrix. }
$$

This is equivalent to saying that the matrix $B$ has a non-zero eigenvalue $(1 / n) \sum_{i=0}^{n} m_{i}$ and $n$ zero eigenvalues, namely, the rank of $B$ is 1, this is also equivalent to saying that $n+1$ row vectors of $B$ are collinear. Equivalently,

$$
\frac{(1 / n) \sum_{i=0}^{n} m_{i}-m_{r}}{\sqrt{m_{r} m_{t}} \cos \varphi_{r t}}=\frac{\sqrt{m_{r} m_{t}} \cos \varphi_{r t}}{(1 / n) \sum_{i=0}^{n} m_{i}-m_{t}}=\frac{\sqrt{m_{r} m_{s}} \cos \varphi_{r s}}{\sqrt{m_{s} m_{t}} \cos \varphi_{s t}}
$$

(where $0 \leq r, s, t \leq n$, and $r, s, t$ are pairwise unequal). Rearranging this, we obtain

$$
\frac{m_{t}}{\sum_{i=0}^{n} m_{i}}=\frac{\cos \varphi_{r s}}{n\left(\cos \varphi_{r s}+\cos \varphi_{r t} \cos \varphi_{s t}\right)} .
$$

Similarly,

$$
\sum_{0 \leq i_{1}<\cdots<i_{k} \leq n}\left(\prod_{1 \leq j \leq k} m_{i_{j}}\right) \sin ^{2} \theta^{\prime}{ }_{i_{1} i_{2} \cdots i_{k}} \leq\left(\frac{1}{n} \sum_{i=1}^{n} m_{i}\right)^{k}\left(\begin{array}{l}
n \\
k
\end{array}\right) .
$$


The equality holds if and only if

$$
\frac{m_{t}}{\sum_{i=0}^{n} m_{i}}=\frac{\cos \varphi_{r s}^{\prime}}{n\left(\cos \varphi_{r s}^{\prime}+\cos \varphi_{r t}^{\prime} \cos \varphi_{s t}^{\prime}\right)}
$$

where $0 \leq r, s, t \leq n$, and $r, s, t$ are pairwise unequal). Using Theorem 3.2 and Cauchy's inequality, we get

$$
\begin{aligned}
& \sum_{0 \leq i_{1}<\cdots<i_{k} \leq n}\left(\prod_{1 \leq j \leq k} m_{i_{j}}\right) \sin ^{2} \widetilde{\theta}_{i_{1} i_{2} \cdots i_{k}} \\
& \leq \sum_{0 \leq i_{1}<\cdots<i_{k} \leq n}\left(\prod_{1 \leq j \leq k} m_{i_{j}}\right) \sin \theta_{i_{1} i_{2} \cdots i_{k}} \sin \theta_{i_{1} i_{2} \cdots i_{k}}^{\prime} \\
& \leq\left[\sum_{0 \leq i_{1}<\cdots<i_{k} \leq n}\left(\prod_{1 \leq j \leq k} m_{i_{j}}\right) \sin ^{2} \theta_{i_{1} i_{2} \cdots i_{k}}\right]^{1 / 2} \\
& \times\left[\sum_{0 \leq i_{1}<\cdots<i_{k} \leq n}\left(\prod_{1 \leq j \leq k} m_{i_{j}}\right) \sin ^{2} \theta^{\prime}{ }_{i_{1} i_{2} \cdots i_{k}}\right]^{1 / 2} \\
& \leq\left(\frac{1}{n} \sum_{i=1}^{n} m_{i}\right)^{k}\left(\begin{array}{l}
n \\
k
\end{array}\right)=\frac{(n-1) !}{k !(n-k) ! n^{k-1}}\left(\sum_{i=1}^{n} m_{i}\right)^{k} .
\end{aligned}
$$

Meanwhile, we obtain that equality (4.4) holds if and only if the hyperplane $\pi_{k} \| \pi_{k^{\prime}}$, $\sin \theta_{i_{1} i_{2} \cdots i_{k}}=c \sin \theta_{i_{1} i_{2} \cdots i_{k}}^{\prime}$ (where $0 \leq c \leq 1$ ), and all the equalities in(4.5) hold.

In particular, when $m_{0}=m_{1}=\cdots=m_{n}$ and $\Omega$ and $\Omega^{\prime}$ are regular, noting $\cos \varphi_{r s}=$ $\cos \varphi_{r s}^{\prime}=(1 / n)(r \neq s),(4.5)$ obviously hold.

From Theorem 4.4, we can easily derive the following interesting inequalities for the mixed vertex angles of two $n$-simplices.

Corollary 5. Let $\widetilde{\theta}_{i_{1} i_{2} \cdots i_{k}}$ be a k-order mixed vertex angle of two $n$-simplices $\Omega$ and $\Omega^{\prime}$, then

$$
\sum_{0 \leq i_{1}<\cdots<i_{k} \leq n} \sin ^{2} \widetilde{\theta}_{i_{1} i_{2} \cdots i_{k}} \leq \frac{n !}{k !(n-k) !}\left(1+\frac{1}{n}\right)^{k}
$$

with equality holds if and only if all the hyperplanes $\pi_{k} \| \pi_{k^{\prime}}, \sin \theta_{i_{1} i_{2} \cdots i_{k}}=c \sin \theta_{i_{1} i_{2} \cdots i_{k}}^{\prime}$ (where $0 \leq c \leq 1$ ), and $\Omega$ and $\Omega^{\prime}$ are regular.

Corollary 6. Let $\widetilde{\varphi}_{i j}$ be the mixed internal dihedral angle between $(n-1)$-dimensional facets $\Omega_{i}$ and $\Omega_{j}^{\prime}$. Then

$$
\sum_{0 \leq i<j \leq n} m_{i} m_{j} \sin ^{2} \widetilde{\varphi}_{i j} \leq \frac{n-1}{2 n}\left(\sum_{i=1}^{n} m_{i}\right)^{2}
$$

with equality holds if and only if all the hyperplanes $\pi_{\left[e_{i}, e_{j}\right]} \| \pi_{\left[e_{i}^{\prime}, e_{j}^{\prime}\right]}, \sin \varphi_{i, j}=c \sin \varphi_{i, j}^{\prime}$, and condition (4.5) holds. 
Corollary 7. Under the conditions of Corollary 6, we have

$$
\sum_{0 \leq i<j \leq n} \sin ^{2} \widetilde{\varphi}_{i j} \leq \frac{(n+1)\left(n^{2}-1\right)}{2 n}
$$

with equality holds if and only if all the hyperplanes $\pi_{\left[e_{i}, e_{j}\right]} \| \pi_{\left[e_{i}^{\prime}, e_{j}^{\prime}\right]}, \sin \varphi_{i, j}=c \sin \varphi_{i, j}^{\prime}$, and $\Omega$ and $\Omega^{\prime}$ are regular.

Corollary 8. Let $\widetilde{\varphi_{i}}(i=0,1, \ldots, n)$ be the mixed vertex angles of $\Omega$ and $\Omega^{\prime}$. Then

$$
\sum_{i=0}^{n} \sin ^{2} \widetilde{\varphi}_{i} \leq\left(1+\frac{1}{n}\right)^{n}
$$

with equality holds if and only if all the hyperplanes $\Omega_{i} \| \Omega_{i}^{\prime}$ and $\Omega$ and $\Omega^{\prime}$ are regular.

\section{Inverse Forms of the Hadamard Inequality}

Let $M=\left(m_{i j}\right)_{n \times n}$ be an $n \times n$ positive Hermitian matrix. The classical Hadamard inequality states that

$$
\operatorname{det} M \leq \prod_{i=1}^{n} m_{i i},
$$

and the equality holds if and only if $M$ is diagonal with entries $m_{i i}$.

Let $\left\{x_{1}, x_{2}, \ldots, x_{n}\right\}$ be a linearly independent vector set from $R^{n}$, and let $V\left(\Pi_{[X]}\right)$ be the $n$-dimensional volume of the $n$-parallelotope $\Pi_{[X]}$ which has $\left\{x_{1}, x_{2}, \ldots, x_{n}\right\}$ as edge-vectors, then the geometric form of the classical Hadamard inequality is as follows:

$$
V\left(\Pi_{[X]}\right) \leq \prod_{i=1}^{n}\left\|x_{i}\right\| .
$$

Let $\Pi_{\left[\hat{x}_{i}\right]}$ be the facet of $\Pi_{[X]}$ which lies in a hyperplane $\pi_{i}$ (i.e., an $(n-1)$-parallelotope which does not contain the vector $x_{i}$ ). Szasz has generalized the Hadamard inequality as follows (see [3]):

$$
V^{n-1}\left(\Pi_{[X]}\right) \leq \prod_{i=1}^{n} V\left(\Pi_{\left[\hat{x}_{i}\right]}\right) .
$$

The equalities in (5.1) and (5.2) occur if and only if $\left\{x_{1}, x_{2}, \cdots, x_{n}\right\}$ is a set of orthogonal non-zero vectors in $R^{n}$.

The other generalizations of the Hadamard inequality to the block matrices and to other types of matrices were obtained by Fisher, Johnson, Markham, Veljan, and others (see [4], [10], [11], and [13]). The estimates for the ratio $\operatorname{det} M / \prod_{i=1}^{n} m_{i i}$ were investigated by Johnson, Newman, Dixon, Reznikov, and others (see [3] and [22]). Some eigenvalues estimates of Wolkowitz and Styan could also be interpreted as estimates of det $M$ (see [25]). 
Let $y_{i}$ be the orthogonal component of $x_{i}$ with respect to $\pi_{i}$. Then we call $y_{i}$ the altitude vector on $\Pi_{\left[\hat{x}_{i}\right]}(i=1,2, \ldots, n)$.

For any linearly independent vector set $\left\{x_{1}, x_{2}, \ldots, x_{n}\right\}$, it is easy to prove that there exists an $n$-parallelotope $\Pi_{[X]}^{*}$ which has $\left\{x_{1}, x_{2}, \ldots, x_{n}\right\}$ as $n$ altitude vectors (see [13]).

In [13] Leng and Zhou proved the inverse forms of the Hadamard inequality and the Szasz inequality as Theorem 5.1 following. In this paper we give a new and simple proof.

Theorem 5.1. Let $\left\{x_{1}, x_{2}, \ldots, x_{n}\right\}$ be a set of linearly independent vectors from $R^{n}$ and let $\Pi_{[X]}^{*}$ be an n-parallelotope which has $\left\{x_{1}, x_{2}, \ldots, x_{n}\right\}$ as the $n$ altitude vectors. Furthermore, let $\Pi_{\left[\hat{x}_{i}\right]}^{*}$ be the $(n-1)$-parallelotope which has $x_{1}, \ldots, x_{i-1}, x_{i+1}, \ldots, x_{n}$ as $(n-1)$ altitude vectors. Then

$$
\begin{aligned}
V\left(\Pi_{[X]}^{*}\right) & \geq \prod_{i=1}^{n}\left\|x_{i}\right\|, \\
V^{n-1}\left(\Pi_{[X]}^{*}\right) & \geq \prod_{i=1}^{n} V\left(\Pi_{\left[\hat{x}_{i}\right]}^{*}\right) .
\end{aligned}
$$

The equalities in (5.3) and (5.4) occur if and only if $\left\{x_{1}, x_{2}, \ldots, x_{n}\right\}$ is a set of orthogonal non-zero vectors in $R^{n}$.

To prove Theorem 5.1, we first prove the following lemma.

Lemma 5.2. Let $X=\left\{x_{1}, x_{2}, \ldots, x_{k}\right\}(k \leq n)$ be a set of linearly independent vectors from $R^{n}$, and let $\Pi_{[X]}$ and $\Pi_{[X]}^{*}$ be as in Theorem 5.1. Then

$$
V\left(\Pi_{[X]}\right) V\left(\Pi_{[X]}^{*}\right)=\left(\prod_{i=1}^{k}\left\|x_{i}\right\|\right)^{2},
$$

where $V\left(\Pi_{[X]}\right)$ and $V\left(\Pi_{[X]}^{*}\right)$ are $k$-dimensional volumes in $R^{n}$.

Proof. Let $Y=\left\{y_{1}, y_{2}, \ldots, y_{k}\right\}$ be the set of $k$ edge-vectors of $\Pi_{[X]}^{*}$, namely, $\Pi_{[X]}^{*}=$ $\Pi_{[Y]}$. According to the definition of $\Pi_{[X]}^{*}$, we have

$$
\left\langle x_{i}, y_{j}\right\rangle=\delta_{i j}\left\|x_{i}\right\|^{2}, \quad \text { where } \quad \delta_{i j}= \begin{cases}1, & i=j, \\ 0, & i \neq j .\end{cases}
$$

It is easy to see that

$$
\begin{gathered}
V_{[X, Y]}^{2}=\left|\operatorname{det}\left(\left\langle x_{i}, y_{j}\right\rangle\right)_{k \times k}\right|=\prod_{i=1}^{k}\left\|x_{i}\right\|^{2}, \\
V\left(\Pi_{[X]}\right) V\left(\Pi_{[X]}^{*}\right)=V_{[X]} V_{[Y]} .
\end{gathered}
$$

Obviously, two vector sets $X=\left\{x_{1}, x_{2}, \ldots, x_{k}\right\}$ and $Y=\left\{y_{1}, y_{2}, \ldots, y_{k}\right\}$ lie in a common $k$-dimensional hyperplane. Using the condition of equality in (2.1), the proof is completed. 
Proof of Theorem 5.1. Taking $k=n$ in Lemma 5.2 and employing inequality (5.1), we obtain

$$
\left(\prod_{i=1}^{n}\left\|x_{i}\right\|\right)^{2}=V\left(\Pi_{[X]}^{*}\right) V\left(\Pi_{[X]}\right) \leq V\left(\Pi_{[X]}^{*}\right) \prod_{i=1}^{n}\left\|x_{i}\right\| .
$$

Rearranging the above inequality, (5.3) is proved.

Taking $k=n-1$ in Lemma 5.2, i.e., applying Lemma 5.2 to set $\left\{x_{1}, \ldots, x_{i-1}, x_{i+1}\right.$, $\left.\ldots, x_{n}\right\}$, we get

$$
V\left(\Pi_{\left[\hat{x}_{i}\right]}^{*}\right) V\left(\Pi_{\left[\hat{x}_{i}\right]}\right)=\prod_{\substack{j=1 \\ j \neq i}}^{n}\left\|x_{i}\right\|^{2}
$$

It follows that

$$
\prod_{i=1}^{n} V\left(\Pi_{\left[\hat{x}_{i}\right]}^{*}\right) V\left(\Pi_{\left[\hat{x}_{i}\right]}\right)=\left(\prod_{i=1}^{n}\left\|x_{i}\right\|\right)^{2(n-1)}=V^{n-1}\left(\Pi_{[X]}^{*}\right) V^{n-1}\left(\Pi_{[X]}\right) .
$$

Applying inequality (5.2), then (5.4) follows.

In succession, we carry on studing the estimation of the volumes of parallelotopes.

For the given $n$-parallelotope $\Pi_{[X]}$ which has $\left\{x_{1}, x_{2}, \ldots, x_{n}\right\}$ as edge vectors, let $\Pi_{\left[\hat{x}_{i}\right]}$ be the facet of $\Pi_{[X]}$ which lies in a hyperplane $\pi_{i}$, let $H=\left\{h_{1}, h_{2}, \ldots, h_{n}\right\}$ be $n$ altitude vectors, and let $\gamma_{i}$ be the angle between edge vector $x_{i}$ and hyperplane $\pi_{i}(1 \leq i \leq n)$. We denote the $n$-parallelotope $\Pi_{[X]}^{\perp}$ which has $h_{1}, h_{2}, \ldots, h_{n}$ as $n$ edge vectors, namely, $\Pi_{[X]}^{\perp}=\Pi_{[H]}$.

Theorem 5.3. Let n-parallelotopes $\Pi_{[X]}$ and $\Pi_{[X]}^{\perp}$ be as above, then

$$
V\left(\Pi_{[X]}\right) V\left(\Pi_{[X]}^{\perp}\right)=\left(\prod_{i=1}^{n}\left\|x_{i}\right\| \sin \gamma_{i}\right)^{2} .
$$

Proof. According to the definition of $\Pi_{[X]}^{\perp}$, we know that $h_{i} \perp \pi_{i}, \quad\left\|h_{i}\right\|=\left\|x_{i}\right\| \sin \gamma_{i}$ $(1 \leq i \leq n)$, and

$$
\left\langle h_{i}, x_{j}\right\rangle=\delta_{i j}\left\|h_{i}\right\|^{2},
$$

where $\delta_{i j}$ is as previously stated.

Similar to the proof of Lemma 5.2,

$$
V\left(\Pi_{[X]}\right) V\left(\Pi_{[X]}^{\perp}\right)=V_{[X, H]}=\prod_{i=1}^{n}\left\|h_{i}\right\|^{2}
$$

and (5.6) follows.

From Theorems 5.1 and 5.3, it is easy to obtain the following result. 
Corollary 9. Under the above hypotheses, we have

$$
V\left(\Pi_{[X]}^{\perp}\right) \leq V\left(\Pi_{[X]}\right) \leq V\left(\Pi_{[X]}^{*}\right) .
$$

The equalities occur if and only if $\left\{x_{1}, x_{2}, \ldots, x_{n}\right\}$ is a set of orthogonal non-zero vectors in $R^{n}$.

To understand the ulterior relations between $\Pi_{[X]}^{*}, \Pi_{[X]}^{\perp}$, and $\Pi_{[X]}$, we define the edge-vertex angle and altitude-vertex angle of an $n$-parallelotope as follows:

Definition 4. Let $h_{i}$ be the altitude vector on $\Pi_{\left[\hat{x}_{i}\right]}(i=1,2, \ldots, n)$ of parallelotope $\Pi_{[X]}$ which has $\left\{x_{1}, x_{2}, \ldots, x_{n}\right\}$ as $n$ edge vectors. Let

$$
\alpha=\arcsin \sqrt{\operatorname{det}\left(\left\langle\frac{x_{i}}{\left\|x_{i}\right\|}, \frac{x_{j}}{\left\|x_{j}\right\|}\right\rangle\right)_{1 \leq i, j \leq n} ;} ;
$$

we call $\alpha$ an edge-vertex angle of the parallelotope $\Pi_{[X]}$.

Let

$$
\beta=\arcsin \sqrt{\operatorname{det}\left(\left\langle\frac{h_{i}}{\left\|h_{i}\right\|}, \frac{h_{j}}{\left\|h_{j}\right\|}\right\rangle\right)_{1 \leq i, j \leq n} ;} ;
$$

we call $\beta$ an altitude-vertex angle of the parallelotope $\Pi_{[X]}$.

\section{Theorem 5.4.}

$$
\operatorname{Sin} \alpha \sin \beta=\prod_{i=1}^{n} \sin \gamma_{i}
$$

Proof. Since

$$
\begin{aligned}
& V\left(\Pi_{[X]}^{\perp}\right)=\sqrt{\operatorname{det}\left(\left\langle h_{i}, h_{j}\right\rangle\right)_{n \times n}}=\sin \beta \prod_{i=1}^{n}\left\|h_{i}\right\|=\sin \beta \prod_{i=1}^{n}\left\|x_{i}\right\| \sin \gamma_{i}, \\
& V\left(\Pi_{[X]}\right)=\sqrt{\operatorname{det}\left(\left\langle x_{i}, x_{j}\right\rangle\right)_{n \times n}}=\sin \alpha \prod_{i=1}^{n}\left\|x_{i}\right\| .
\end{aligned}
$$

According to Theorem 5.3, the proof is completed.

Theorem 5.5. Under the above hypotheses, then

$$
\begin{aligned}
& \frac{V\left(\Pi_{[X]}\right)}{V\left(\Pi_{[X]}^{*}\right)}=\sin ^{2} \alpha, \\
& \frac{V\left(\Pi_{[X]}^{\perp}\right)}{V\left(\Pi_{[X]}\right)}=\sin ^{2} \beta, \\
& \frac{V\left(\Pi_{[X]}^{\perp}\right)}{V\left(\Pi_{[X]}^{*}\right)}=\sin ^{2} \alpha \sin ^{2} \beta .
\end{aligned}
$$


Proof. According to the definition of $V\left(\Pi_{[X]}\right)$, we have that

$$
V\left(\Pi_{[X]}\right)^{2}=\operatorname{det}\left(\left\langle x_{i}, x_{j}\right\rangle\right)_{n \times n}=\sin ^{2} \alpha \prod_{i=1}^{n}\left\|x_{i}\right\|^{2} .
$$

Combining this with equality (5.5) in Lemma 5.2, equality (5.9) follows.

Using Theorems 5.3 and 5.4, we get that

$$
V\left(\Pi_{[X]}^{\perp}\right) V\left(\Pi_{[X]}\right)=\sin ^{2} \alpha \sin ^{2} \beta \prod_{i=1}^{n}\left\|x_{i}\right\|^{2}=\sin ^{2} \beta V\left(\Pi_{[X]}\right)^{2}
$$

Rearranging it, equality (5.10) follows.

Combining (5.9) with (5.10), we get (5.11).

At last, we study the relationship of $V\left(\Pi_{[X]} \cup \Pi_{[Y]}\right)$ and $V_{[X, Y]}$. In [16] Leng et al. introduce the concept of a parallelotope-stack.

Let $\Pi_{[X]}$ and $\Pi_{[Y]}$ be two k-parallelotopes in $R^{n}$. The union $\Pi_{[X]} \cup \Pi_{[Y]}$ is called the parallelotope-stack of $\Pi_{[X]}$ and $\Pi_{[Y]}$ if there is a hyperplane $H$ such that the two parallelotopes lie in opposite half-spaces bounded by $H$ and $H$ contains a facet of each.

Theorem 5.6. Let $X=\left\{x_{1}, x_{2}, \ldots, x_{k}\right\}$ and $Y=\left\{y_{1}, y_{2}, \ldots, y_{k}\right\}$ be two sets of linearly independent vectors, let $\Pi_{[X]}$ and $\Pi_{[Y]}$ be the $k$-parallelotopes which takes $X$ and $Y$ as $k$ edge vectors, respectively. Suppose that $\Pi_{[X]} \cup \Pi_{[Y]}$ is the parallelotope-stack. Then

$$
V_{[X, Y]} \leq \frac{1}{2} V\left(\Pi_{[X]} \cup \Pi_{[Y]}\right)
$$

Proof. According to the definition of parallelotope-stack $\Pi_{[X]} \cup \Pi_{[Y]}$ of $\Pi_{[X]}$ and $\Pi_{[Y]}$ in $R^{n}$, we infer

$$
V\left(\Pi_{[X]} \cup \Pi_{[Y]}\right)=V\left(\Pi_{[X]}\right)+V\left(\Pi_{[Y]}\right) .
$$

On the other hand, it is easy to see that

$$
V\left(\Pi_{[X]}\right)=V_{[X]}, \quad V\left(\Pi_{[Y]}\right)=V_{[Y]} .
$$

Applying Theorem 1, we obtain that

$$
V_{[X]}+V_{[Y]} \geq 2 \sqrt{V_{[X]} V_{[Y]}} \geq 2 V_{[X, Y]}
$$

and this implies (5.12).

\section{Acknowledgements}

The authors are greatly indebted to Professor Hanfang Zhang for his help. 


\section{References}

1. P. Bartoš, Sinusova veta o simplexoch $v E_{n}$, Časopis Pěst. Mat., 93 (1968), 273-277.

2. L. M. Blumenthal, Theory and Applications of Distance Geometry, Oxford University Press, London, 1953.

3. J. D. Dixon, How good is Hadamard's inequality for determinants?, Canad. Math. Bull., 27 (1984), 260-264.

4. G. M. Engle and H. Schneider, The Hadamard-Fischer inequality for a class of matrices defined by eigenvalue monotonicity, Linear and Multilinear Algebra, 4 (1976), 155-176.

5. F. Eriksson, The law of sines for tetrahedra and $n$-simplices, Geom. Dedicata, 7 (1978), 71-80.

6. R. J. Gardner, Intersection bodies and the Busemann-Petty problem, Trans. Amer. Math. Soc., 342 (1994), 435-445.

7. R. J. Gardner, Geometric Tomography, Cambridge University Press, Cambridge, 1995.

8. S. G. Guo, Generalization of Bartoš volume formulas for high-dimensional simplex, J. Math. Res. Exposition, 18 (1998), 597-600.

9. R. Horn and C. R. Johnson, Matrix Analysis, Cambridge University Press, New York, 1985.

10. C. R. Johnson and T. Markham, Compression and Hadamard power inequalities for matrices, Linear and Multilinear Algebra, 18 (1985), 23-34.

11. C. R. Johnson and M. Newman, How bad is the Hadamard determinantal bound?, J. Res. Nat. Bur. Standards, 78 (1974), 167-169.

12. G. S. Leng and Y. Zhang, The generalized sine theorem and inequalities for simplices, Linear Algebra and its Applications, 278 (1998), 237-247.

13. G. S. Leng, Inverse forms of Hadamard inequality, SIAM J. Matrix Anal. Appl., 23(4) (2002), 990-997.

14. G. S. Leng, Z. Shen, and L. H. Tang, Inequalities for two simplices, J. Math. Anal. Appl., 248 (2000), $429-437$.

15. G. S. Leng and L. H. Tang, Some generalizations to several dimensional of Pedoe inequality with applications, Acta. Math. Sinica, 40 (1997), 14-21 (in Chinese).

16. G. S. Leng, Y. Zhang, and B. L. Ma, Largest parallelotopes contained in simplices, Discrete Math., 211 (2000), 111-123.

17. E. Lutwak, Dual mixed volumes, Pacific J. Math., 58 (1975), 531-538.

18. E. Lutwak, Volume of mixed bodies, Trans. Amer. Math. Soc., 294 (1986), 487-500.

19. E. Lutwak, Centroid bodies and dual mixed volumes, Proc. London Math. Soc., 60 (1990), 365-391.

20. D. Pedoe, An inequality for two triangles, Math. Proc. Cambridge Philos. Soc., 38 (1942), 397-398.

21. D. Pedoe, Thinking geometrically, Amer. Math. Monthly, 77 (1970), 711-721.

22. A. G. Reznikov, Determinant inequalities with applications to isoperimetrical inequalities, Oper. Theory Adv. Appl., 77 (1995), 139-244.

23. R. Schneider, Convex Bodies: The Brunn-Minkoeski Theory, Cambridge University Press, Cambridge, 1993.

24. D. Veljan, The sine theorem and inequalities for volumes of simplices and determinants, Linear Algebra Appl., 219 (1995), 79-91.

25. H. Wolkowitz and G. Styan, Bounds for eigenvalues using traces, Linear Algebra Appl., 29 (1980), $471-$ 506.

26. D. H. Yang, The Geometric relation involving $k$-dimensional vertex angle of two simplicies and its applications (to appear).

27. G. Y. Zhang, Centered bodies and dual mixed volumes, Trans. Amer. Math. Soc., 345 (1994), 777-801.

28. L. Yang and J. Z. Zhang, A generalization to several dimensions of the Neuberg-Pedoe inequality with applications, Bull. Austral. Math. Soc., 27 (1983), 203-214.

29. Y. Zhang, A conjection for the volume of simplex with feet of perpendicular as vertices, J. Systems Sci. Math. Sci., 12 (1992), 371-375.

Received July 5, 2003, and in revised form December 7, 2003. Online publication June 7, 2004. 